\title{
Phonon Scattering through a Local Anisotropic Structural Disorder in the Thermoelectric Solid Solution $\mathrm{Cu}_{2} \mathrm{Zn}_{1-x} \mathrm{Fe}_{x} \mathrm{GeSe}_{4}$
}

Wolfgang G. Zeier, ${ }^{\dagger, \ddagger}$ Yanzhong Pei, ${ }^{\ddagger}$ Gregory Pomrehn, ${ }^{\ddagger}$ Tristan Day, ${ }^{\ddagger}$ Nicholas Heinz, Christophe P. Heinrich, ${ }^{\dagger}$ G. Jeffrey Snyder, ${ }^{* *}$ and Wolfgang Tremel ${ }^{* \dagger}$

${ }^{\dagger}$ Institut für Anorganische Chemie und Analytische Chemie der Johannes Gutenberg-Universität, Duesbergweg 10-14, D-55099 Mainz, Germany

${ }^{\ddagger}$ Material Science, California Institute of Technology, Pasadena, California 91125, United States

\section{Supporting Information}

ABSTRACT: Inspired by the promising thermoelectric properties of chalcopyrite-like quaternary chalcogenides, here we describe the synthesis and characterization of the solid solution $\mathrm{Cu}_{2} \mathrm{Zn}_{1-x} \mathrm{Fe}_{x} \mathrm{GeSe}_{4}$. Upon substitution of $\mathrm{Zn}$ with the isoelectronic $\mathrm{Fe}$, no charge carriers are introduced in these intrinsic semiconductors. However, a change in lattice parameters, expressed in an elongation of the $c / a$ lattice parameter ratio with minimal change in unit cell volume, reveals the existence of a three-stage cation restructuring process of $\mathrm{Cu}, \mathrm{Zn}$, and $\mathrm{Fe}$. The resulting local anisotropic structural disorder leads to phonon scattering not normally observed, resulting in an effective approach to reduce the lattice thermal conductivity in this class of materials.

\section{INTRODUCTION}

Quaternary chalcogenides have recently been investigated as promising candidates for thermoelectric power generation. ${ }^{1-5}$ Thermoelectric materials can convert thermal energy into electrical energy, opening promising possible applications for waste heat recovery or other specialized applications. ${ }^{6,7}$ The performance of a thermoelectric material is given by its figure of merit $z T$, with $z T=\alpha^{2} T / \rho \kappa$, where a high Seebeck coefficient $\alpha$, low electrical resistivity $\rho$, and low thermal conductivity $\kappa$ are necessary to obtain high thermoelectric efficiencies.

Different strategies to obtain low thermal conductivity for a high thermoelectric efficiency include utilization of the materials' structure, for example, use of filling atoms in skutterudites, ${ }^{8-10}$ or by using nanostructuring to reduce the phonon mean free path. ${ }^{11-15}$ Another effective way to decrease the thermal conductivity of a material can be achieved via point defect scattering through alloying with other elements, ${ }^{16}$ successfully shown for thermoelectric materials in Heusler compounds, ${ }^{17}$ skutterudites, ${ }^{18,19}$ lead telluride, ${ }^{20}$ and SiGe. ${ }^{21,22}$ While alloying with isoelectronic elements does not introduce charge carriers into a material, it creates point defect scattering for phonons due to mass differences ${ }^{23}$ (or mass field fluctuations) and strain field fluctuations. ${ }^{17}$ This strain arises from different bonding interactions and size differences between the host and impurity atoms, ${ }^{17}$ and expresses itself in the lattice parameters.

Inspired by the reduction of the lattice thermal conductivities of the quaternary compounds $\mathrm{Cu}_{2} \mathrm{ZnGeSe}_{4}$ and $\mathrm{Cu}_{2} \mathrm{CdSnSe}_{4}$ via phase segregation ${ }^{5}$ and nano crystals, ${ }^{24,25}$ we have investigated the effect of substitution of $\mathrm{Zn}$ with isoelectronic
Fe in the solid solution $\mathrm{Cu}_{2} \mathrm{Zn}_{1-x} \mathrm{Fe}_{x} \mathrm{GeSe}_{4}$ on the thermal transport properties. As expected, alloying with an isoelectronic element does not introduce charge carriers. However, a change in lattice parameters is observed, which does not follow Vegards' law. An elongation of the lattice due to a three-stage restructuring process of the cations $\mathrm{Cu}^{+}, \mathrm{Zn}^{2+}$, and $\mathrm{Fe}^{2+}$ leads to a high local anisotropic structural disorder and therefore a statistically significant reduction of the lattice thermal conductivity by $\sim 15 \%$ due to point defect scattering.

\section{EXPERIMENTAL SECTION}

Synthesis. Bulk samples of polycrystalline $\mathrm{Cu}_{2} \mathrm{Zn}_{1-x} \mathrm{Fe}_{x} \mathrm{GeSe}_{4}$ with compositions of $x=0,0.1, \ldots, 0.9,1.0$ were prepared via solid-state reactions using elemental powders of $\mathrm{Cu}$ (Alfa Aesar, 99.999\%), Zn (Sigma Aldrich, 99.995\%), Fe (Alfa Aesar, 99.998\%), Ge (Chempur, 99.99\%), and Se (Alfa Aesar, 99.999\%). Phase purity of the starting materials was verified by X-ray diffraction, and all synthetic procedures were carried out in a $\mathrm{N}_{2}$ drybox. Annealing was performed in evacuated quartz ampules, which were preheated at $1073 \mathrm{~K}$ under dynamic vacuum for $5 \mathrm{~h}$ to ensure dry conditions.

The starting elements were thoroughly ground, sealed in quartz ampules, and annealed in a first step for $48 \mathrm{~h}$ at 923 and $673 \mathrm{~K}$, for $x<$ 0.4 and $x \geq 0.4$, respectively. In a following second step, the harvested powders were reannealed for $96 \mathrm{~h}$ at 1073 and $873 \mathrm{~K}$, respectively. Heating and cooling rates for all procedures in the horizontal tube furnaces were $5 \mathrm{~K} / \mathrm{min}$. It was found that the second annealing step was necessary to prevent the formation of the binary and ternary compositions. The difference in the heat treatments was necessary to

Received: August 30, 2012

Published: December 20, 2012 
prevent the decomposition of the Fe-containing compounds. The quartz ampules were $10-12 \mathrm{~cm}$ in length and $11 \mathrm{~mm}$ inner diameter with a maximum amount of $1.5 \mathrm{~g}$ of starting materials within the ampule. This ampule geometry was found to prevent significant loss of selenium at higher temperatures, indicated by red selenium precipitation present in longer ampules. The obtained powders were hand ground and consolidated into $1-1.5 \mathrm{~mm}$ thick, $12 \mathrm{~mm}$ diameter disks at $873 \mathrm{~K}$ for $5 \mathrm{~h}$ under a pressure of $40 \mathrm{MPa}$ by induction hot pressing in high density graphite dies. ${ }^{26}$ The resulting samples have more than $95 \%$ theoretical density, determined from the mass and geometry of the consolidated disks.

Physical Characterization. X-ray diffraction measurements were performed on a Siemens D5000 powder diffractometer with a Braun M50 position sensitive detector and $\mathrm{Cu} \mathrm{K} \alpha \alpha_{1}$ radiation (Ge (220) monochromator) with a step size of $0.0078^{\circ}$ in $2 \Theta$. Pawley refinements were performed with TOPAS Academic V4.1 ${ }^{27}$ applying the fundamental parameter approach using the crystallographic data from Schäfer and Nitsche. ${ }^{28}$ The instrumental measurement uncertainty for the determination of the lattice parameters is approximated to be $0.003 \AA$.

Thermal diffusivity was measured using a Netzsch laser flash diffusivity instrument (LFA 457), and samples were coated with a thin layer of graphite to minimize errors in the emissivity. The data were analyzed using a Cowan model with pulse correction. Heat capacity was estimated using the method of Dulong-Petit, and theoretical densities were calculated from the molar mass and the lattice parameters for each composition obtained from X-ray diffraction. The Seebeck coefficient was calculated from the slope of the thermopower versus temperature gradient measurements from Chromel-Nb thermocouples, applying a temperature gradient of 10 $\mathrm{K}^{29}$ Electrical resistivity, Hall coefficient, and carrier concentration were measured using the Van der Pauw technique under a reversible magnetic field of $2 \mathrm{~T}$, a current of $20 \mathrm{~mA}$, and pressure-assisted contacts. All measurements were performed under dynamic vacuum and on multiple samples for each composition. Shown measurement data show the data collected during heating and cooling.

Density Functional Calculations. Density functional calculations were performed with the WIEN2K code ${ }^{30}$ based on the full-potential linearized augmented plane-wave (FP-LAPW) method under the generalized gradient approximation (GGA) as parametrized by Perdew, Burke, and Ernzerhof (PBE). ${ }^{31}$ A plane-wave basis cutoff was $R_{\mathrm{mt}} K_{\max }=7$, in terms of the smallest muffin tin radius and maximum plane wave vector respectively. Muffin tin radii for $\mathrm{Cu}, \mathrm{Zn}$, $\mathrm{Fe}, \mathrm{Ge}$, and Se were 2.4, 2,5, 2.4, 2.3, and 2.1 au, respectively. Calculations were performed at the theoretical ground-state lattice parameters as determined by a structural minimization of the unit cell. Atomic positions were relaxed to converged forces below $0.025 \mathrm{eV} /$ angstrom on each atom. An antiferromagnetic state was used for the initial magnetization of the density. Total energy convergence was achieved in a self-consistent calculation using a shifted $8 \times 8 \times 4 \mathrm{k}$ point mesh, which reduced to 32 symmetrically unique k-points. The modified Becke-Johnson (TB-mBJ) ${ }^{32}$ semilocal exchange potential was employed, which has been shown to improve the band gap across a wide variety of semiconductors, ${ }^{33}$ but may still fall short in these materials. This method was utilized to produce the density of states and electronic dispersions shown in this work.

\section{RESULTS AND DISCUSSION}

Chemical Characterization. All samples were checked for phase purity prior to any transport measurements, and complete powder diffraction data are shown in the Supporting Information for each composition. In all compositions, all reflections can be indexed to $\mathrm{Cu}_{2} \mathrm{Zn}_{1-x} \mathrm{Fe}_{x} \mathrm{GeSe}_{4}$, and no secondary phases are observed. Refined lattice parameters are shown in Table 1. Scanning electron microscopy confirms phase purity and similar microstructures in the whole composition range (for representative images, see the Supporting Information). Powder X-ray diffraction data of
Table 1. Lattice Parameters $a, c, V, c / a$-Ratio, and Profile Residuals $R_{\text {wp }}$ for $\mathrm{Cu}_{2} \mathrm{Zn}_{1-x} \mathrm{Fe}_{x} \mathrm{GeSe}_{4}$, from the Corresponding Pawley Refinement

\begin{tabular}{cccccc}
$x$ & $a / \AA$ & $c / \AA$ & $V / \AA^{3}$ & $c / a$ & $R_{w p}$ \\
0.0 & $5.6121(2)$ & $11.0480(2)$ & 347.96 & 1.969 & 6.6 \\
0.1 & $5.6080(3)$ & $11.0536(2)$ & 347.64 & 1.971 & 4.5 \\
0.2 & $5.6036(2)$ & $11.0661(1)$ & 347.48 & 1.975 & 4.4 \\
0.3 & $5.6079(9)$ & $11.0935(3)$ & 348.88 & 1.978 & 4.2 \\
0.4 & $5.6022(6)$ & $11.1046(3)$ & 348.52 & 1.982 & 4.3 \\
0.5 & $5.5879(6)$ & $11.1025(1)$ & 346.68 & 1.987 & 3.8 \\
0.6 & $5.5901(5)$ & $11.1122(1)$ & 347.25 & 1.988 & 3.9 \\
0.7 & $5.5827(2)$ & $11.1176(2)$ & 346.50 & 1.991 & 3.8 \\
0.8 & $5.5918(2)$ & $11.1076(1)$ & 347.32 & 1.986 & 3.6 \\
0.9 & $5.5956(0)$ & $11.0924(8)$ & 346.84 & 1.980 & 4.0 \\
1.0 & $5.6014(6)$ & $11.0703(8)$ & 347.35 & 1.976 & 5.7 \\
\hline
\end{tabular}

samples prior to the hot pressing procedure and data taken from consolidated samples do not show any significant texture in this material. Therefore, properties measured on disks of this polycrystalline material are expected to be isotropic within expected experimental uncertainty and represent a scalar average of the tensor properties.

Pawley refinements of the powder X-ray diffraction data reveal a change in lattice parameters upon substitution of $\mathrm{Zn}$ with $\mathrm{Fe}$ as seen in Figure 1. Experimental $c / a$-ratios (see Figure

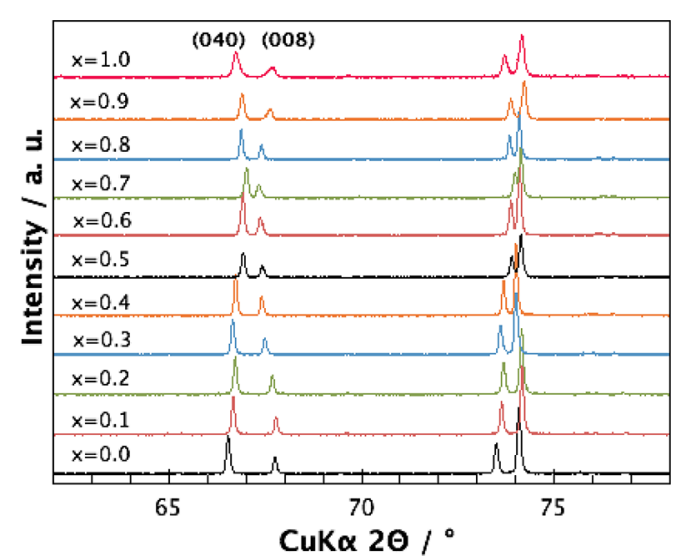

Figure 1. Reflections of selected Miller indices (040) and (008), showing the change in lattice parameters upon substitution. These Miller planes do not show a monotonous change with $x$ and do not cross over linearly, as one would expect in a solid solution that follows Vegard's law.

2) exhibit a non linear trend, showing an elongation of the $c$ axis with increasing $\mathrm{Fe}$ content until a maximum is reached at $70 \% \mathrm{Fe}$ content, where this trend becomes reversed. This nonVegard like behavior has been observed by Caldera et al.; however, they have assumed it to be not significant giving experimental uncertainty. ${ }^{34}$ In this work, the lattice parameters change significantly as compared to the measurement uncertainty; furthermore, this discontinuity of the lattice parameters has also previously been observed in the solid solution $\mathrm{Cu}_{2} \mathrm{Fe}_{1-x} \mathrm{Zn}_{x} \mathrm{SnS}_{4}{ }^{35-37}$ A phase segregation due to the solubility limit being reached has been ruled out, because no splitting of reflections at higher diffraction angles is observed (see Figure 1). Furthermore, ${ }^{57} \mathrm{Fe}$ Mössbauer spectroscopy on this class of compounds shows the existence of $\mathrm{Fe}^{2+}$ only, therefore ruling out the influence of $\mathrm{Fe}^{3+}$ on the crystal structure. $^{38}$ 


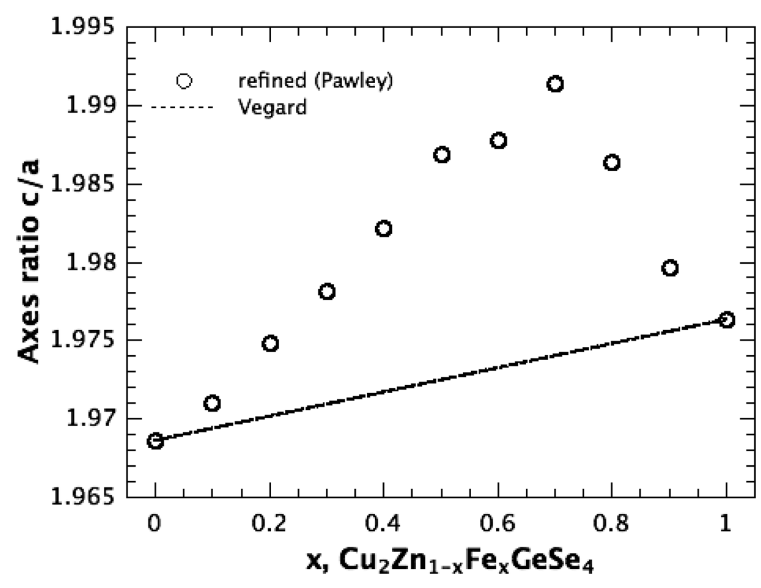

Figure 2. Lattice parameter $c / a$-ratio with composition $x$ of $\mathrm{Cu}_{2} \mathrm{Zn}_{1-x} \mathrm{Fe}_{x} \mathrm{GeSe}_{4}$, from a Pawley refinement. The broken line indicates the expected behavior following Vegard's law. A three-stage cation restructuring process of $\mathrm{Cu}^{+}, \mathrm{Fe}^{2+}$, and $\mathrm{Zn}^{2+}$ results in the trend of the lattice parameters.

Neutron diffraction studies by Schorr et al..$^{39,40}$ on the chemically and structurally very similar solid solution $\mathrm{Cu}_{2} \mathrm{Fe}_{1-x} \mathrm{Zn}_{x} \mathrm{SnS}_{4}$ reported a similar behavior of the lattice parameters and the $c / a$-ratio upon substitution, which can be described as a three-stage cation restructuring process in the crossover from the stannite $\left(\mathrm{Cu}_{2} \mathrm{ZnGeSe}_{4}\right.$, space group $\left.I \overline{4} 2 m\right)$ to kesterite $\left(\mathrm{Cu}_{2} \mathrm{FeGeSe}_{4}\right.$, space group $\left.I \overline{4}\right)$ structure type (see Figure 3). ${ }^{39,40}$ Upon substitution of $\mathrm{Zn}$, Fe starts to replace $\mathrm{Zn}$ on the $2 a$-site $(0,0,0)$ as expected (Figure $3 b)$. In the range of $0.35<x<0.7$, which is the main restructuring region, $\mathrm{Cu}$ depletes the $4 d$-site $(0,1 / 2,1 / 4)$, occupying the $2 a$-site and forcing $\mathrm{Zn}$ on the $4 d$-site, while Fe holds its position $(2 a){ }^{39,40}$ At a substitution level of $x=0.7$, the cations $\mathrm{Fe}^{2+}, \mathrm{Zn}^{2+}$, and $\mathrm{Cu}^{+}$are equally distributed on the $2 a$-site ((Figure $3 \mathrm{c}$ ) and $\mathrm{Cu}$ and $\mathrm{Zn}$ on position $4 d$, exhibiting the highest $c / a$-ratio and highest local anisotropic structural disorder of this solid solution. With further decreasing $\mathrm{Zn}$ content, $\mathrm{Cu}$ starts to dominate position $2 a$ and $\mathrm{Fe}$ and $\mathrm{Cu}$ occupy position $4 d$ in $\mathrm{Cu}_{2} \mathrm{FeGeSe}_{4}$ (Figure 3d). ${ }^{39,40}$ The differences in bonding interaction between the cations and the anions lead to different bond lengths and bond angles, resulting in the observed trend in the lattice parameters. ${ }^{40}$

Electronic Structure Results. The electronic dispersion along lines of high symmetry from the DFT calculations is shown in Figure 4 for $\mathrm{Cu}_{2} \mathrm{ZnGeSe}_{4}$ in the stannite structure and $\mathrm{Cu}_{2} \mathrm{FeGeSe}_{4}$ in the kesterite structure. Both materials exhibit direct band gaps at the Brillouin zone center. The stannite

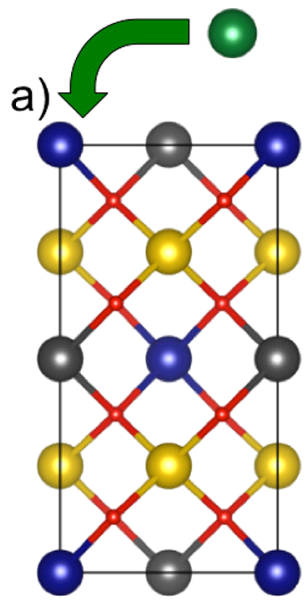

b)

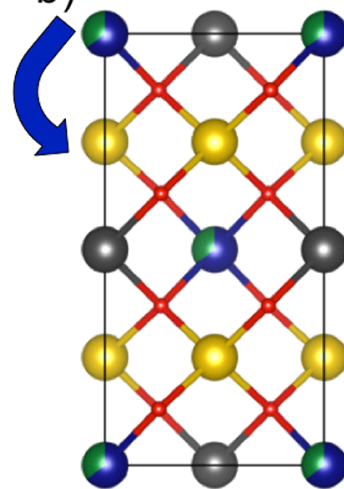

c)

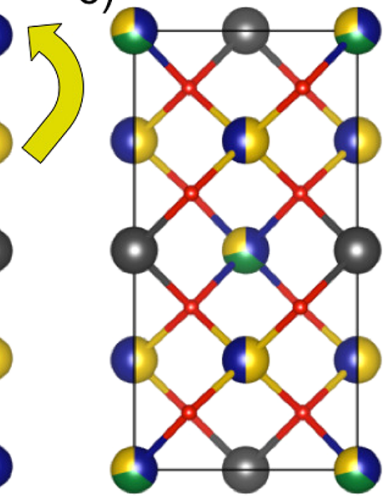

d)

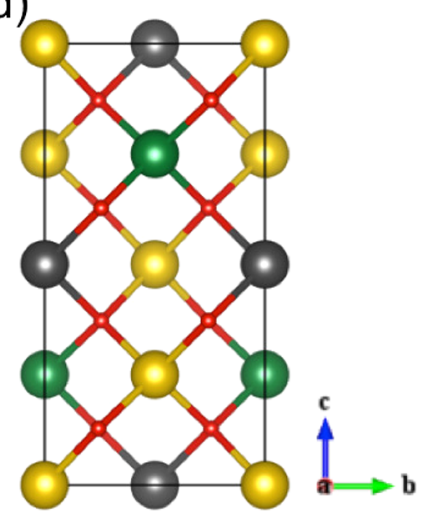

e)

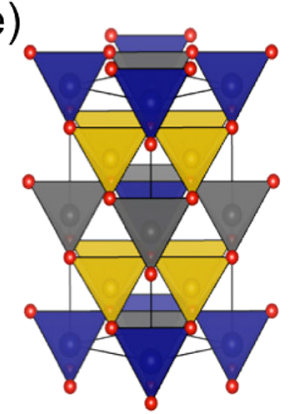

f)

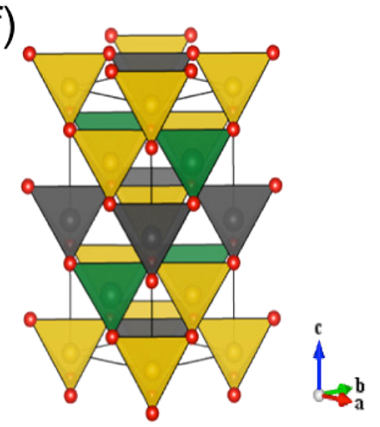

Figure 3. Top: Three-stage cation restructuring process from the stannite $\left(\mathrm{Cu}_{2} \mathrm{ZnGeSe}_{4}, \mathrm{a}\right)$ to kesterite $\left(\mathrm{Cu}_{2} \mathrm{FeGeSe}_{4}, \mathrm{~d}\right)$ structure type. Cu atoms are yellow, Ge atoms gray, $\mathrm{Zn}$ atoms are blue, Fe atoms green, and $\mathrm{Se}$ atoms are red. The sizes of the atoms in this ball-and-stick model are arbitrary and have been chosen for a better structural representation. With increasing Fe content, Fe starts to replace $\mathrm{Zn}(\mathrm{a})$. Cu then depletes its original site and occupies the $\mathrm{Zn}$ site, while forcing $\mathrm{Zn}$ on the $\mathrm{Cu}$ site, which results in the kesterite structure (d) type with further decreasing $\mathrm{Zn}$ content. The arrows represent the substitution and reordering of $\mathrm{Zn}$ with $\mathrm{Fe}$ from (a) to (d). Bottom: Crystal structures of stannite $\mathrm{Cu}_{2} \mathrm{ZnGeSe}_{4}$ (e) and kesterite $\mathrm{Cu}_{2} \mathrm{FeGeSe}_{4}$ (f) in polyhedral representation with the tetrahedral coordination of the elements indicated. Note the difference in the occupation of the different specific lattice sites of $\mathrm{Cu}$ and the $\mathrm{M}^{\mathrm{II}}$ cations. 


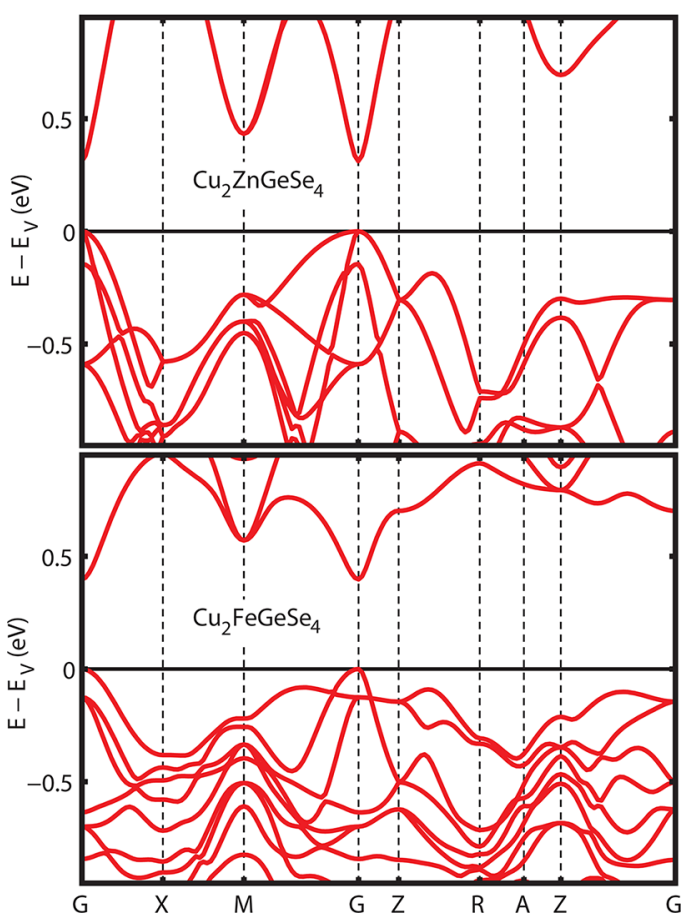

Figure 4. Calculated band structures of stannite-type $\mathrm{Cu}_{2} \mathrm{ZnGeSe}_{4}$ (top) and kesterite-type $\mathrm{Cu}_{2} \mathrm{FeGeSe}_{4}$ (bottom). Note the presence of a heavy band, primarily of $\mathrm{Fe}$ d-state character, in the valence band of $\mathrm{Cu}_{2} \mathrm{FeGeSe}_{4}$, which is expected to contribute to the transport properties.

structured $\mathrm{Cu}_{2} \mathrm{ZnGeSe}_{4}$ exhibits a degeneracy of two at the valence band maximum. The two bands split apart away from the gamma point, and each exhibit parabolic behavior with averaged effective mass of $0.81 m_{\mathrm{e}}$ and $0.23 \mathrm{~m}_{\mathrm{e}}$, respectively. The top valence band of $\mathrm{Cu}_{2} \mathrm{FeGeSe}_{4}$ in the kesterite structure is a single band with parabolic band mass of $0.67 \mathrm{~m}_{\mathrm{e}}$. Around 82 $\mathrm{meV}$ below the valence band maximum, there is another band with predominantly Fe d-state character. This band has a much heavier effective mass of $1.91 \mathrm{~m}_{\mathrm{e}}$ and a symmetric degeneracy, $N_{\mathrm{V}}$, of four. It is expected that this band would contribute to electronic transport due to its proximity to the valence band maximum.

Transport Properties. The temperature dependence of the electrical resistivity $\rho$ for different compositions $x$ of $\mathrm{Cu}_{2} \mathrm{Zn}_{1-x} \mathrm{Fe}_{x} \mathrm{GeSe}_{4}$ is shown in Figure 5. As expected for these wide band gap semiconductors, ${ }^{5}$ these compositions exhibit a high electrical resistivity at room temperature, decreasing with temperature. A previously reported phase transformation $^{5}$ is apparent for $x=0.0$ and 0.1 . No charge carriers are introduced directly through the substitution with isoelectronic $\mathrm{Fe}^{2+}$ for $\mathrm{Zn}^{2+}$; however, measured Hall carrier concentrations (see Figure 6) change across compositions due to the changes in character of the density of states of the valence band (see Figure 4) as well as the presence of intrinsic defects. The resistivity of a composition is therefore mainly controlled by the change in mobility, due to alloy scattering, and charge carrier concentrations. The unchanged intrinsic nature of these semiconductors, upon substitution of $\mathrm{Zn}$ with $\mathrm{Fe}$, can also be seen in the temperature-dependent positive Seebeck coefficients (Figure 7), which show intrinsic p-type conduction. ${ }^{5,41}$ The differences in the Seebeck coefficients arise from the different carrier concentrations of the samples and a likely increase in the carrier effective mass with the presence of Fe. The differences in the valley degeneracy of the stannite and kesterite type may also influence the Seebeck coefficients. This effect is still under investigation.

The total thermal conductivity was calculated from the measured thermal diffusivity using $\kappa=D d C_{p}$, where $D=$ thermal diffusivity, $d=$ geometric density, and $C_{p}=$ specific heat capacity. Here, use of the Dulong-Petit approximation for the heat capacity is likely to result in an underestimation $(\sim 10 \%)$ of the thermal conductivity at high temperatures. ${ }^{42}$ The temperature dependence of the total thermal conductivity is shown in Figure 8 , and the effect of the previously mentioned phase transformation can be seen at around $450 \mathrm{~K}$. It has to be emphasized that the atomic structure for the thermal conductivity calculations described below is guided by the structure of the room temperature phase and not the unknown high temperature phase.

The lattice thermal conductivity $\kappa_{\text {lattice }}$ at room temperature (see Figure 9) was evaluated by subtracting the electronic contribution $\left(\kappa_{\mathrm{el}}=L T \rho^{-1}\right.$, where $L=1.5 \times 10^{-8} \mathrm{~W} \Omega \mathrm{K}^{-2}$ for non degenerate materials ${ }^{43}$ ), that is, $\kappa_{\text {lattice }}=\kappa-L T \rho^{-1}$. While the measurement uncertainty for the laser flash diffusivity measurement is $3 \%$, we have assumed a combined uncertainty for $\kappa_{\text {lattice }}$ of $5 \%$ at room temperature, taking into account a $5 \%$ error for the resistivity measurement. Upon substitution with $\mathrm{Fe}$, the lattice thermal conductivity decreases with increasing $\mathrm{Fe}$ content until a minimum value is reached at $70 \% \mathrm{Fe}$ content, where this trend reverses. Because the mass difference between
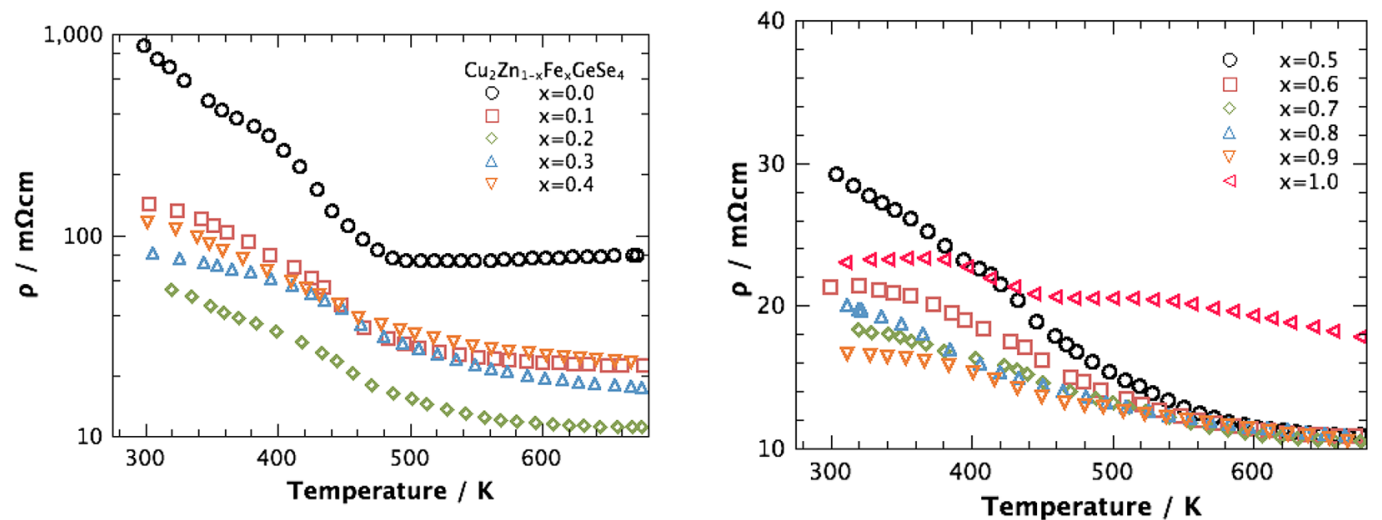

Figure 5. Temperature dependence of the electrical resistivity $\rho$ of $\mathrm{Cu}_{2} \mathrm{Zn}_{1-x} \mathrm{Fe}_{x} \mathrm{GeSe}_{4}$ for $x=0.0-0.4$ (left) and $x=0.5-1.0$ (right) showing semiconducting behavior with high electrical resistivity. A previously reported phase transformation ${ }^{5}$ is apparent for $x=0.0$ and 0.1 . 

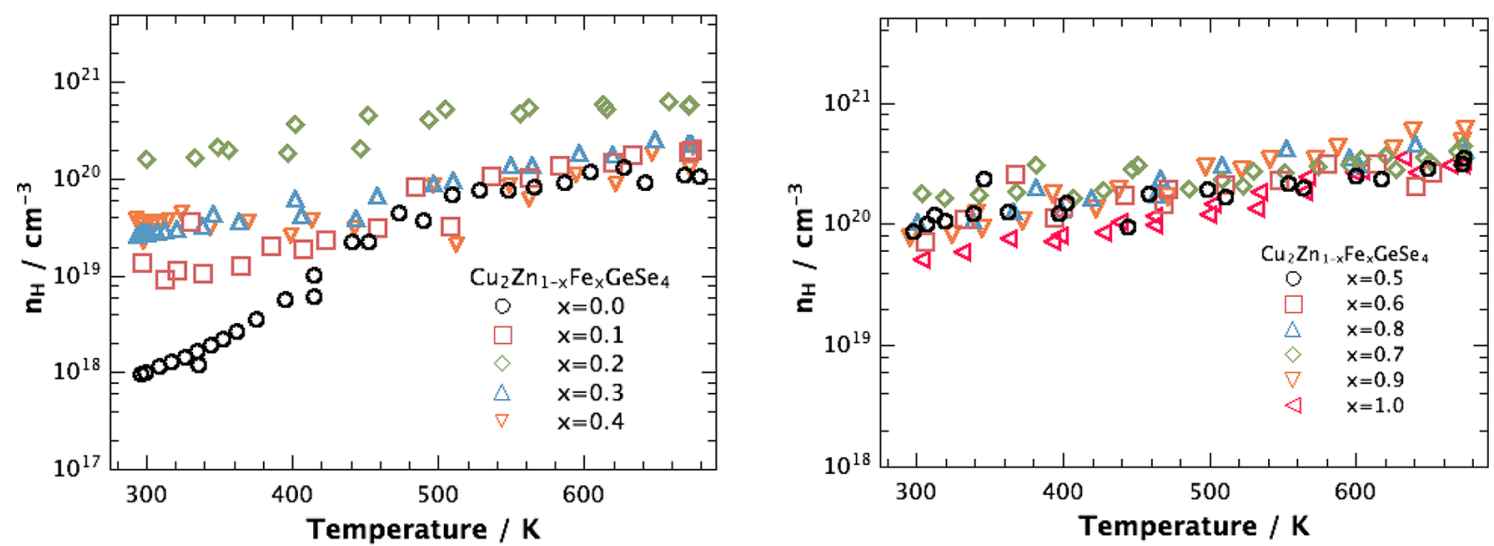

Figure 6. Temperature-dependent Hall carrier concentrations $n_{\mathrm{H}}$ of $\mathrm{Cu}_{2} \mathrm{Zn}_{1-x} \mathrm{Fe}_{x} \mathrm{GeSe}_{4}$ for $x=0.0-0.4$ (left) and $x=0.5-1.0$ (right). There is no trend of charge carrier concentrations with composition apparent, as expected for isoelectronic doping with the presence of intrinsic defects.
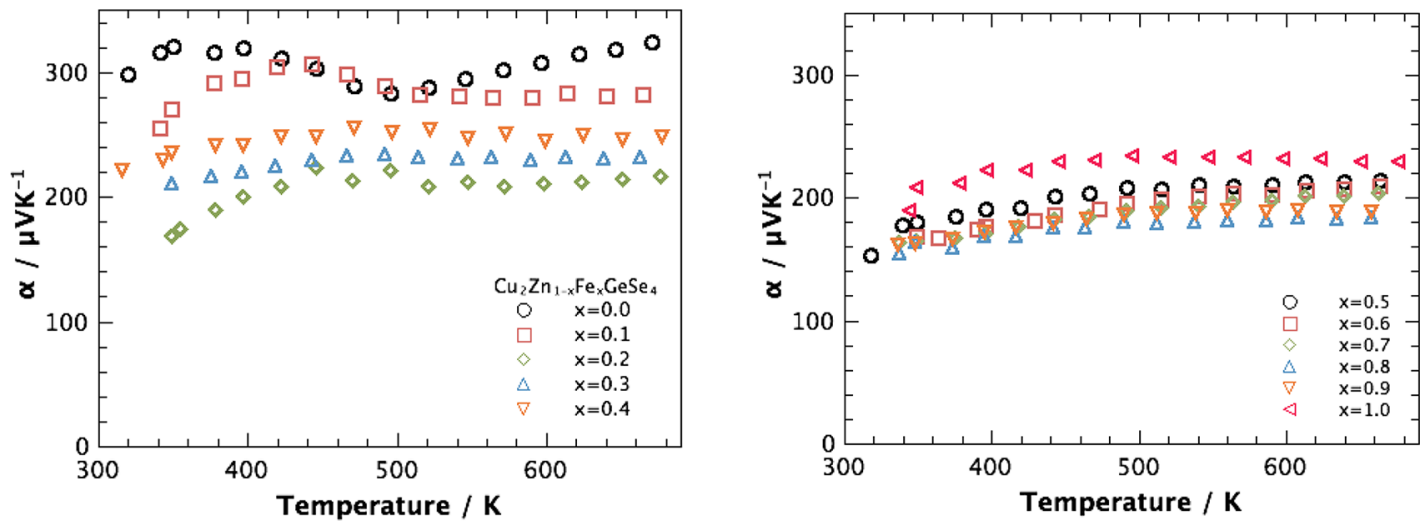

Figure 7. Temperature dependence of the Seebeck coefficients $\alpha$ of $\mathrm{Cu}_{2} \mathrm{Zn}_{1-x} \mathrm{Fe}_{x} \mathrm{GeSe}_{4}$ for $x=0.0-0.4$ (left) and $x=0.5-1.0$ (right) showing intrinsic p-type semiconducting behavior controlled by intrinsic defects. A previously reported phase transformation ${ }^{5}$ is apparent for $x=0.0$ and 0.1 .
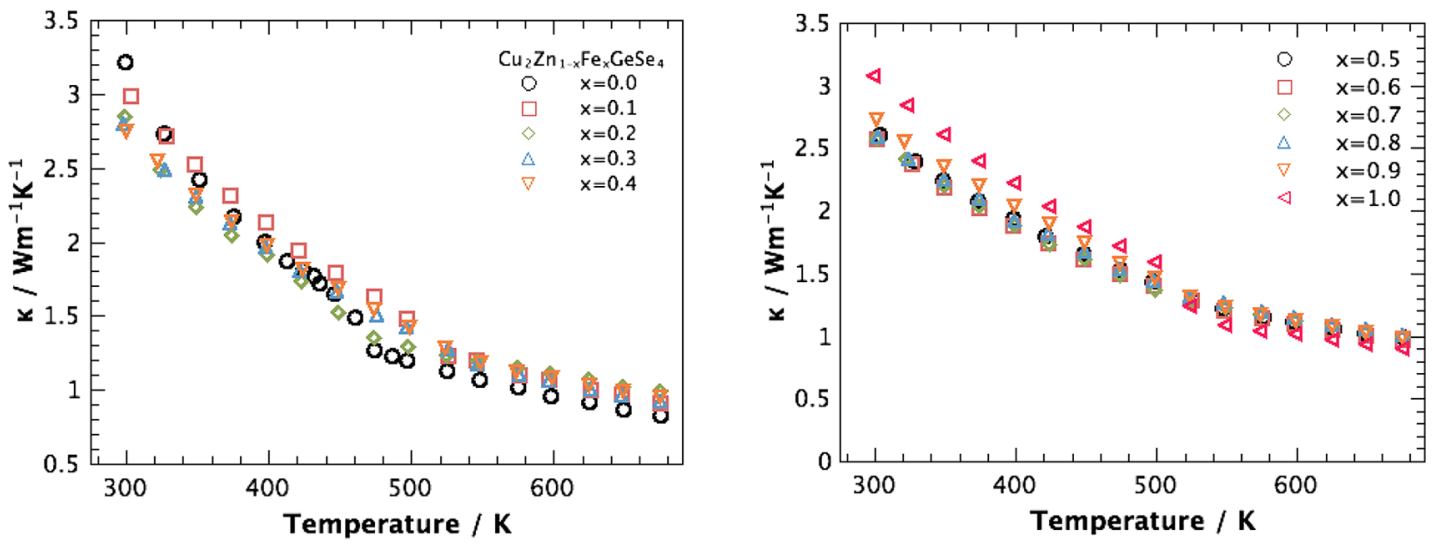

Figure 8. Total thermal conductivity $\kappa$ of $\mathrm{Cu}_{2} \mathrm{Zn}_{1-x} \mathrm{Fe}_{x} \mathrm{GeSe}_{4}$ for $x=0.0-0.4$ (left) and $x=0.5-1.0$ (right), decreasing with temperature due to Umklapp-scattering processes.

Fe and $\mathrm{Zn}$ is small, this statistically significant reduction of the lattice thermal conductivity cannot be explained by the mass contrast only, as indicated in Figure 9, where the expected $\kappa_{\text {lattice }}$ has been calculated within the Callaway model. ${ }^{16,17,20}$ Furthermore, effects of the microstructure can be ruled out because similar microstructures and no impurity phases are apparent in the SEM micrographs.

The structural change that correlates with this dramatic reduction in thermal conductivity at $70 \% \mathrm{Fe}$ is the three-stage cation restructuring process described in Figure 3 where the highest local anisotropic structural disorder occurs at at 70\% Fe content. This disorder apparently reduces the lattice thermal conductivity by $15 \%$, resulting in a minimum for the composition $\mathrm{Cu}_{2} \mathrm{Zn}_{0.3} \mathrm{Fe}_{0.7} \mathrm{GeSe}_{4}$ due to scattering of phonons by local anisotropic strain. As seen in Figures 8 and 9, the reduction of the lattice thermal conductivity by point defect scattering is more effective at lower temperatures. With increasing temperatures, the high frequency phonons get scattered mainly by phonon-phonon processes reducing their phonon mean free path. In other words, the dominant 


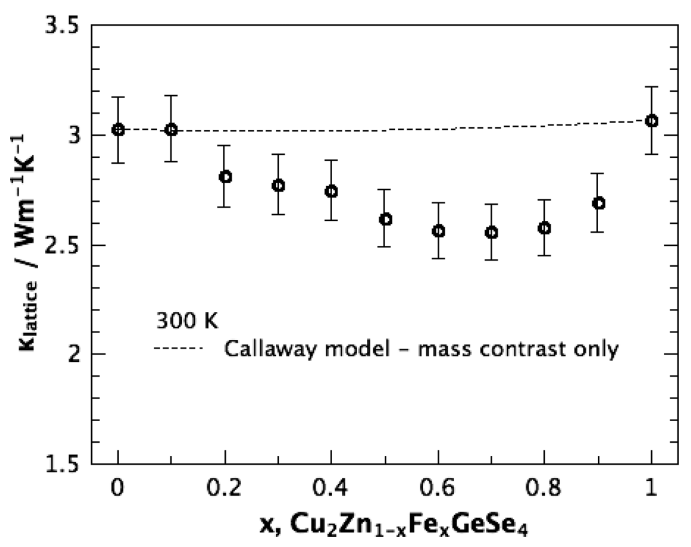

Figure 9. Lattice thermal conductivity $\kappa_{\text {lattice }}$ of $\mathrm{Cu}_{2} \mathrm{Zn}_{1-x} \mathrm{Fe}_{x} \mathrm{GeSe}_{4}$ at room temperature showing a $15 \%$ reduction due to disorder scattering. An error of 5\% is assumed for $\kappa_{\text {lattice }}$ at room temperature. The broken line indicates the calculated influence of the mass contrast between $\mathrm{Fe}$ and $\mathrm{Zn}$ on the lattice thermal conductivity, which shows that the strain related to the lattice constant change has a significant effect on the lattice thermal conductivity.

scattering mechanism at higher temperatures is phononphonon Umklapp scattering, and point defect scattering does not contribute hugely to the lattice thermal conductivity. ${ }^{44}$

\section{CONCLUSION}

In summary, we have shown the dramatic effect on the lattice thermal conductivity due to the substitution of isoelectronic and similar sized $\mathrm{Fe}$ for $\mathrm{Zn}$ in the solid solution $\mathrm{Cu}_{2} \mathrm{Zn}_{1-x} \mathrm{Fe}_{x} \mathrm{GeSe}_{4}$. While the intrinsic electronic nature of the material is not changed upon substitution with $\mathrm{Fe}^{2+}$, a distinct change in the lattice parameter $c / a$-ratio has been observed, which can be explained by a three-stage cation restructuring and disordering process of $\mathrm{Fe}, \mathrm{Zn}$, and $\mathrm{Cu}$. The restructuring leads to a reduction of the lattice thermal conductivity by $15 \%$ at $70 \% \mathrm{Fe}$ content. As the disorder scattering of the phonons, due to mass fluctuation and isotropic strain, is expected to be very small from these similar mass and similar volume ions, this work shows the potential of significant point defect scattering due to local anisotropic strain that also results in a change in $c / a$-ratio.

\section{ASSOCIATED CONTENT}

\section{S Supporting Information}

Representative scanning electron micrographs for $x=0.3,0.5$, 0.7 , and 1.0 of $\mathrm{Cu}_{2} \mathrm{Zn}_{1-x} \mathrm{Fe}_{x} \mathrm{GeSe}_{4}$ are shown in Figure S1. Figures $\mathrm{S} 1-\mathrm{S} 12$ show complete $\mathrm{X}$-ray diffraction data for all compositions including profile fits, profile differences, and TICS from the corresponding Pawley refinements. This material is available free of charge via the Internet at http:// pubs.acs.org.

\section{AUTHOR INFORMATION}

\section{Corresponding Author}

jsnyder@caltech.edu; tremel@uni-mainz.de

Notes

The authors declare no competing financial interest.

\section{ACKNOWLEDGMENTS}

Financial support through the Excellence Initiative (DFG/GSC 266) is acknowledged by W.G.Z. and C.P.H. W.G.Z. furthermore thanks the Carl-Zeiss foundation for financial support. We also acknowledge Vadim Ksenofontov for performing Mössbauer spectroscopy.

\section{REFERENCES}

(1) Shi, X. Y.; Huang, F. Q.; Liu, M. L.; Chen, L. D. Appl. Phys. Lett. 2009, 94, 122103.

(2) Liu, M.-L.; Chen, I.-W.; Huang, F.-Q.; Chen, L.-D. Adv. Mater. 2009, 21, 3808-3812.

(3) Liu, M.-L.; Huang, F.-Q.; Chen, L.-D.; Chen, I.-W. Appl. Phys. Lett. 2009, 94, 202103.

(4) Sevik, C. Appl. Phys. Lett. 2009, 95, 112105.

(5) Zeier, W. G.; Lalonde, A.; Gibbs, Z. M.; Heinrich, C. P.; Panthöfer, M.; Snyder, G. J.; Tremel, W. J. Am. Chem. Soc. 2012, 137, $7147-7154$.

(6) Bell, L. E. Science 2008, 321, 1457-1461.

(7) DiSalvo, F. J. Science 1999, 285, 703-706.

(8) Sales, B.; Mandrus, D.; Williams, R. Science 1996, 272, 13251328.

(9) Nolas, G. S.; Poon, J.; Kanatzidis, M. G. MRS Bull. 2006, 31, 199-205.

(10) Shi, X.; Yang, J.; Salvador, J. R.; Chi, M.; Cho, J. Y.; Wang, H.; Bai, S.; Yang, J.; Zhang, W.; Chen, L. J. Am. Chem. Soc. 2011, 133, 7837-7846.

(11) Pei, Y.; Heinz, N. A.; LaLonde, A.; Snyder, G. J. Energy Environ. Sci. 2011, 4, 3640.

(12) Pei, Y.; Lensch-Falk, J.; Toberer, E. S.; Medlin, D. L.; Snyder, G. J. Adv. Funct. Mater. 2011, 241-249.

(13) Johnsen, S.; He, J.; Androulakis, J.; Dravid, V. P.; Todorov, I.; Chung, D. Y.; Kanatzidis, M. G. J. Am. Chem. Soc. 2011, 133, 34603470.

(14) Minnich, A. J.; Dresselhaus, M. S.; Ren, Z. F.; Chen, G. Energy Environ. Sci. 2009, 2, 466-479.

(15) Poudeu, P. F. P.; D’Angelo, J.; Downey, A. D.; Short, J. L.; Hogan, T. P.; Kanatzidis, M. G. Angew. Chem., Int. Ed. 2006, 45, 3835-3839.

(16) Callaway, J.; Baeyer, H. C. Phys. Rev. 1960, 126, 1149-1154.

(17) Yang, J.; Meisner, G. P.; Chen, L. Appl. Phys. Lett. 2004, 85, $1140-1142$

(18) Meisner, G.; Morelli, D.; Hu, S.; Yang, J.; Uher, C. Phys. Rev. Lett. 1998, 80, 3551-3554.

(19) Zhou, Z.; Uher, C.; Jewell, A.; Caillat, T. Phys. Rev. B 2005, 71, 235209.

(20) Alekseeva, G. T.; Efimova, B. A.; Ostrovskaya, L. M.; Serebryannikova, O. S.; Tsypin, M. I. Sov. Phys.-Semiconductors 1971, 4, 1122-1125.

(21) Abeles, B. Phys. Rev. 1963, 131, 1906-1911.

(22) Steigmeier, E.; Abeles, B. Phys. Rev. 1964, 136, 1149-1155.

(23) Ambegaokar, V. Phys. Rev. 1959, 114, 488-489.

(24) Ibáñez, M.; Cadavid, D.; Zamani, R.; García-Castello, N.; Izquierdo-Roca, V.; Li, W.; Fairbrother, A.; Prades, J. D.; Shavel, A.; Arbiol, J.; Perez-Rodriguez, A.; Morante, J. R.; Cabot, A. Chem. Mater. 2012, 24, 562-570.

(25) Ibáñez, M.; Zamani, R.; LaLonde, A.; Cadavid, D.; Li, W.; Shavel, A.; Arbiol, J.; Morante, J. R.; Gorsse, S.; Snyder, G. J.; Cabot, A. J. Am. Chem. Soc. 2012, 134, 4060-4063.

(26) LaLonde, A. D.; Ikeda, T.; Snyder, G. J. Rev. Sci. Instrum. 2011 82,025104

(27) Cohelo, A. TOPAS Academic V4.1, 2004.

(28) Schäfer, W.; Nitsche, R. Mater. Res. Bull. 1974, 9, 645-654.

(29) Iwanaga, S.; Toberer, E. S.; LaLonde, A.; Snyder, G. J. Rev. Sci. Instrum. 2011, 82, 063905.

(30) Blaha, P.; Schwarz, K.; Madsen, G.; Kvasnicka, D.; Luitz, J. WIEN2k: An Augmented Plane Wave plus Local Orbitals Program for Calculating Crystal Properties; Institute of Physical and Theoretical Chemistry: TU Vienna, 2001.

(31) Perdew, J. P.; Burke, K.; Ernzerhof, M. Phys. Rev. Lett. 1996, 77, 3865-3868. 
(32) Tran, F.; Blaha, P. Phys. Rev. Lett. 2009, 102, 226401.

(33) Koller, D.; Tran, F.; Blaha, P. Phys. Rev. B 2011, 83, 195134.

(34) Caldera, D.; Quintero, M.; Morocoima, M.; Quintero, E.; Grima, P.; Marchan, N.; Moreno, E.; Bocaranda, P.; Delgado, G.; Mora, A.; Briceño, J.; Fernandez, J. J. Alloys Compd. 2008, 457, 221224.

(35) Hall, S. R.; Ssymanski, J. T.; Stewart, J. M. Can. Mineral. 1978, $16,131-137$.

(36) Bonazzi, P.; Bindi, L.; Bernardini, G. P.; Menchetti, S. Can. Mineral. 2003, 41, 639-647.

(37) Bernardini, G. P.; Bonazzi, P.; Corazza, M.; Corsini, F.; Mazzetti, G.; Poggi, L.; Tanelli, G. Eur. J. Mineral. 1990, 2, 219-225.

(38) Elbschütz, M.; Hermon, E.; Shtrikman. J. Phys. Chem. Solids 1967, 28, 1633-1636.

(39) Schorr, S.; Hoebler, H.-J.; Tovar, M. Eur. J. Mineral. 2007, 19, 65-73.

(40) Schorr, S. Thin Solid Films 2007, 515, 5985-5991.

(41) Porras, A.; Quintero, M.; Barrios, R.; Gonzalez, J.; Tovar, R. Phys. Status Solidi B 1999, 1067, 1067-1073.

(42) Delaire, O.; May, A. F.; McGuire, M. A.; Porter, W. D.; Lucas, M. S.; Stone, M. B.; Abernathy, D. L.; Ravi, V. A.; Firdosy, S. A.; Snyder, G. J. Phys. Rev. B 2009, 80, 184302.

(43) Goldsmid, H. J. Applications of Thermoelectricity; Butler \& Tanner Ltd.: London, 1960.

(44) Toberer, E. S.; May, A. F.; Melot, B. C.; Flage-Larsen, E.; Snyder, G. J. Dalton Trans. 2010, 39, 1046-1054. 